Wioletta A. Piegzik

Uniwersytet Szczeciński https://orcid.org/0000-0002-6552-6236 wioletta.piegzik@usz.edu.pl

\title{
ÉVALUER LES CONNAISSANCES IMPLICITES ET EXPLICITES EN FRANÇAIS LANGUE ÉTRANGÈRE PAR LES TESTS DE JUGEMENT DE GRAMMATICALITÉ : ANALYSE DES OUTILS ET PRÉSENTATION DES RÉSULTATS DE LA RECHERCHE
}

\author{
Evaluation of implicit and explicit knowledge in French as a foreign \\ language through grammatical judgment tests: analysis of tools \\ and presentation of research results
}

The aim of this paper is to evaluate implicit and explicit knowledge among Polish speaking students studying French as a foreign language in the academic environment. In the first part, we discuss a timed grammaticality judgment test (TGJT) as an instrument for measuring implicit knowledge and an untimed grammaticality judgment test (UGJT) as a tool for evaluating explicit knowledge and we propose solutions to sidestep some of the weaknesses of both these instruments which were observed in our research. The second part focuses on the presentation of the research results, leading to the conclusion that the group of students at $A 2$ level develop implicit and explicit knowledge in a fairly balanced way.

Keywords: implicit knowledge of a foreign language, explicit knowledge of a foreign language, French as a foreign language, timed grammaticality judgment test, untimed grammaticality judgment test

Słowa kluczowe: wiedza implicytna w JO, wiedza eksplicytna w JO, język francuski jako obcy, test oceny poprawności gramatycznej oparty na limitowanym czasie, test oceny poprawności gramatycznej bez limitu czasu 


\section{Introduction}

Dans le présent article, on discute les tests qui ont été élaborés pour évaluer les connaissances implicites et explicites en grammaire de français langue étrangère des étudiants polonophones représentant le niveau de compétences A2. On s'efforce de présenter plus précisément la spécificité des outils dont la construction s'appuie sur une distinction bien connue en psychologie cognitive, à savoir connaissances implicites/connaissances explicites. La description proposée mettra l'accent sur les qualités des outils choisis, ainsi que sur leurs déficiences. En s'appuyant sur les propres recherches de l'auteur, réalisées dans le milieu académique où se déroule la formation des futurs professionnels en langue, on tâchera également de proposer quelques solutions visant à surmonter les insuffisances éprouvées afin d'élaborer des outils plus pertinents et plus valables.

La mesure des connaissances implicites et explicites en langue étrangère effectuée par les tests de jugement de grammaticalité joue un grand rôle dans la compréhension de la spécificité des opérations mentales mises en œuvre pendant l'apprentissage-acquisition d'une langue par les locuteurs adultes étudiant celle-ci dans un contexte formel. En effet, la mesure dont on parle donne accès à deux types d'opérations mentales différentes qui fondent l'apprentissage-acquisition. D'un côté, il s'agit des opérations mentales se passant à l'insu de son locuteur, et de l'autre côté, on a affaire au traitement engageant la pensée consciente et la réflexion métalinguistique qui en découle. L'accès à ces deux types d'opérations mentales, s'opérant sur le même matériel linguistique, apporte de nouveaux acquis sur la manière dont le locuteur adulte développe sa compétence de communication. Le sujet abordé semble être d'autant plus pertinent qu'il concerne les apprenants dont les compétences en langue ne sont pas avancées. La mesure de leurs connaissances implicites en grammaire se caractérise en premier lieu par la spontanéité et le caractère procédural, alors que leurs connaissances explicites, liées à la métaréflexion, semblent suggérer que le groupe d'utilisateurs de langue au niveau débutant développe parallèlement les connaissances fondant l'aisance de la prise de parole et les connaissances assurant le contrôle et la prise de conscience en langue cible. Ceci est plutôt nouveau, parce que l'on admet, généralement, que dans le contexte d'apprentissage formel ce sont les connaissances explicites qui se développent en premier pour finir par se transformer, à force de pratique linguistique et communicative relativement longue et systématique, en connaissances assurant la spontanéité de l'expression orale (Ellis 2009, DeKeyser 2007).

Ainsi, la première partie du présent article sera focalisée sur la présentation des deux outils utilisés pour mesurer les connaissances implicites et 
explicites des étudiants du FLE, à savoir le test de jugement de grammaticalité en temps limité et le test de jugement de grammaticalité en temps illimité. La seconde partie de l'article présentera les résultats de la recherche menée et conduira à vérifier l'hypothèse selon laquelle l'enseignement de la grammaire dans le cadre des études universitaires philologiques favorise le développement des deux types de connaissances - explicites et implicites.

\section{Les outils servant à mesurer les connaissances implicites et explicites en langue}

Pour juger les connaissances implicites en langue, on a choisi le test de jugement de grammaticalité en temps limité (TJGTL) ; et pour juger les connaissances explicites, on s'est servi de son correspondant qui exclut la pression du temps, c'est-à-dire du test de jugement de grammaticalité en temps illimité (TJGTI). Les deux tests, basés sur le même objectif (mesurer les connaissances en langue) et s'opérant sur le même matériel langagier (les mêmes phrases), s'appuient pourtant sur différents modes de pensée. Cette différence semble avoir une importance capitale pour comprendre deux comportements mentaux opposés mais aussi parfois complémentaires.

\subsection{Le test de jugement de grammaticalité en temps limité}

Le test de jugement de grammaticalité en temps limité renvoie en premier lieu aux intuitions que les utilisateurs d'une langue possèdent et avec lesquelles ils sont capables d'évaluer les phrases du point de vue de leur conformité aux règles de grammaire. Les intuitions sont comprises ici par nous dans l'acception de sentiments qui s'imposent immédiatement dans l'esprit du locuteur et se caractérisent par la sûreté et la facilité avec lesquelles ils ont été construits. La compréhension de l'intuition ici avancée renvoie, en grande partie, à celle qui a été proposée par Noam Chomsky dans son ouvrage Aspects of the Theory of Syntax $(1965)^{1}$ où le linguiste américain percevait les intuitions comme un élément inhérent à la compétence linguistique qui permet au locuteur de formuler des jugements d'acceptabilité sur des phrases dans sa L1. Elle a aussi des points communs avec l'intuition heuristique définie par Samir Bajrić (2005 : 16) en tant qu'«appréhension implicite du système grammaticale » qui permet aux locuteurs d'être « intimement convaincus que telle

\footnotetext{
1 Il est important de noter que l'acception de l'intuition de Chomsky remontant aux années 60. détermine jusqu'à aujourd'hui la représentation que beaucoup de chercheurs en linguistique possèdent sur l'intuition en langue (voir p.ex. Yaguello 1988: 155, Dubois 2012: 257 ou Polański 2003: 261).
} 
phrase est grammaticalement inattaquable ou, au contraire, qu'elle est litigieuse, sans pouvoir dire pourquoi ". En pratique, le locuteur juge qu'une phrase est grammaticale alors qu' une autre ne respecte pas les règles sans pour autant être capable d'en présenter d'explication ni d'explicitation. Il s'agit alors d'opérations mentales sensées qui échappent à la conscience et au contrôle. Le TJGTL a donc pour objectif de saisir les intuitions que les apprenants ont déjà acquises dans une langue cible et qui constituent la base de leurs comportements langagiers spontanés. Dans le comportement langagier choisi pour tester les connaissances des apprenants d'une langue étrangère (prendre vite une décision concernant la correction grammaticale), on voit l'analogie avec le comportement langagier des locuteurs natifs qui savent, démontrant ainsi leurs connaissances implicites élevées, trancher immédiatement et avec certitude la grammaticalité/agrammaticalité des phrases proposées. Cela constitue pour certains linguistes (comme Chomsky), le meilleur moyen de comprendre comment le locuteur prend les décisions concernant la forme, mais aussi comment la langue fonctionne dans l'esprit de son locuteur.

Shawn Loewen (2009 : 94) définit le TJGTL comme une tâche (ang. task) qui consiste à décider si une phrase est grammaticale ou si elle ne l'est pas. Un élément capital sur lequel le TJGTL est fondé, comme l'indique d'ailleurs son nom, est la limitation du temps. On admet que le temps prévu pour juger une phrase devrait être très bref, parce que la pression du temps correspond à la nature des connaissances implicites qui se caractérisent avant tout par la vitesse avec laquelle elles sont accessibles au locuteur, ce qui renvoie, en grande partie, à leur caractère intuitif. Dans les ouvrages consacrés à l'acquisition des langues, on note une absence de consensus parmi les chercheurs sur un temps exact destiné à la réponse. Certains accordent aux participants de la recherche émettant le jugement de grammaticalité d'une phrase de longueur moyenne (de 5 à 10 syllabes) un temps limité à 3-3,5 secondes (Han 2000), tandis que d'autres prolongent le temps en admettant que le traitement d'information en langue étrangère, surtout lorsque le niveau de maîtrise n'est pas trop élevé, dure plus longtemps. Ce désaccord constitue certainement la source des objections soulevées autour du TJGTL et peut remettre en cause la validité de cet instrument de mesure. Toutefois, il est évident que pour avoir la certitude ${ }^{2}$ que l'on n'a accès qu'aux connaissances implicites et non pas à un mélange de connaissances implicites et explicites, il est indispensable d'offrir aux participants un temps similaire à celui dont ont besoin les

\footnotetext{
${ }^{2}$ Le terme 'certitude' n'a pas la même signification en sciences humaines que dans les sciences basées sur des algorithmes et se servant de lois très strictes vérifiables dans chaque situation.
} 
locuteurs natifs pour répondre à la même question. II semble aussi important de proposer des phrases qui soient claires et compréhensibles, c'est-à-dire qui correspondent au niveau des participants afin qu'ils puissent saisir la structure lorsque le sens véhiculé est immédiatement décrypté et ne soulève pas de doutes.

Il convient de remarquer que le premier grand avantage du TJGTL est qu'il permet de vérifier facilement et rapidement les constructions grammaticales qui intéressent le chercheur et qui pourraient être difficiles à saisir dans la production libre des participants. II s'agit, généralement, des structures grammaticales considérées comme essentielles pour la langue étudiée, c'est-à-dire celles qui sont fréquemment utilisées par les locuteurs natifs et/ou celles qui reflètent la singularité de cette langue. En examinant la connaissance des structures grammaticales, le TJGTL vérifie, comme l'observe d'ailleurs Loewen (2009 : 94), les habilités morphosyntaxiques des sujets de la recherche. En effet, les tests présentés dans la littérature spécialisée embrassent uniquement l'examen du niveau morphologique et syntaxique de la langue. Cependant, en tenant compte de la spécificité de ce test, on est d'avis qu'il pourrait également servir à mesurer les habilités phonétiques et phonologiques.

La facilité au niveau des instructions est un autre avantage du test en question. La consigne donnée aux participants est particulièrement simple parce qu'elle renvoie seulement à une seule activité : marquer la phrase jugée comme correcte. En pratique, il s'agit soit d'appuyer sur un bouton (lorsque les phrases sont affichées successivement sur l'écran d'un ordinateur ou d'un projecteur), soit de mettre des croix sur un formulaire papier (lorsque les phrases sont enregistrées, puis diffusées avec un strict décalage correspondant au temps prévu pour chaque réponse).

Si le chercheur tient au caractère formateur du test et non pas seulement à la collecte de données, il est possible que la participation au TJGTL favorise la prise de conscience et l'autocorrection qui en découle. Dans ce cas-là, il s'agit du TJGTL dans sa version binaire. Elle consiste en la présentation d'un doublet de phrases dont l'une est correcte et l'autre viole une règle de grammaire. II est fort possible que la confrontation offerte aux participants (grammatical-agrammatical) constitue une source importante de " court-circuit ", en complémentarité avec le traitement simultané et intuitif qui mène, par conséquent, à l'autocorrection. Le point de vue évoqué reste conforme à l'approche socio-constructiviste appliquée à la didactique des langues (Nadeau et Fisher 2011) et à la pédagogie (Barth 2002), mettant en valeur l'exploitation du contraste des exemples positifs et négatifs comme un moyen puissant servant à cerner la forme correcte par le rejet de celle qui ne respecte pas la norme de langue. Dans ce cas-là, le statut de l'erreur change : elle se révèle être un outil conduisant à la prise de conscience et aux défossilisations, c'està-dire au déracinement des fautes tenaces commises par tout apprenant. 
Le plus souvent, les phrases incorrectes sont celles qui viennent de la pratique du chercheur qui est aussi souvent enseignant de langue. Ainsi, on se réfère aux fautes les plus fréquentes pour une communauté linguistique donnée et à celles qui relèvent de la projection des structures de la $L 1$ sur celles de la $L 2$.

Malgré ses qualités, décrites ci-dessus, le TJGTL présente aussi des limites importantes.

Une première contrainte qui affecte l'outil en question est le fait que certains apprenants participant à la recherche peuvent éprouver une gêne psychique, voire un stress lié à la pression inhérente à la limitation du temps. Cette tension résulte certainement du fait que les apprenants ne sont pas habitués aux outils qui limitent le temps pour chaque réponse. Les tests " standards " s'appuient généralement sur une limitation qui concerne l'ensemble des exercices à réaliser où chaque apprenant est libre et gère le temps en fonction de ses besoins individuels. À notre avis, un court entraînement aux réponses rapides, précédant le TJGTL, semble remédier à cette insuffisance. L'expérience issue de la recherche montre que les sujets sont capables d'adopter très rapidement ce comportement. Finalement, après avoir terminé leurs tests, ils expriment souvent la satisfaction éprouvée à l'idée d'avoir su agir dans un temps limité à l'instar des locuteurs natifs et avancés.

La possibilité de répondre au hasard constitue une autre limite du TJGTL. Il est impossible d'exclure que les participants, en jugeant la grammaticalité des phrases, ne marquent de réponses dont ils ne soient pas sûrs, mais qu'ils ont tout simplement devinées. Le TJGTL partage donc ce défaut avec les tests à choix multiples ou avec la technique du "vrai-faux ". Notre solution, mise en pratique pendant la recherche menée auprès d'étudiants en FLE, est de laisser aux participants la possibilité de marquer, en cas de doute ou de manque de réponse spontanée, un point d'interrogation. En nous appuyant sur notre expérience, nous pouvons constater que cette solution s'avère, au moins dans certains cas, efficace. La solution proposée ne résout pas complètement la déficience observée, mais la diminue néanmoins.

Enfin, il est important de remarquer que le TJGTL ne vérifie que des habilités réceptives. La capacité à juger la grammaticalité des phrases permet au chercheur de tirer des conclusions importantes portant sur la manière dont les apprenants réagissent linguistiquement, mais elle ne mesure que l'une des habilités faisant partie des connaissances implicites. Pour disposer d'une image plus complète, il est donc nécessaire d'associer cet outil à d'autres servant à mesurer les capacités productives. Dans la littérature spécialisée, on cite par exemple le test de narration oral (ang. oral narrative test) (Ellis 2009; Kim 2010) avec lequel le chercheur peut évaluer les capacités de construction des phrases, de structuration du récit et de prononciation correcte des sons d'une L2. 
Évaluer les connaissances implicites et explicites en français langue étrangère par...

Dans le TJGTL mené ici (annexe 1$)^{3}$, les étudiants en FLE disposaient de 3 secondes pour chaque réponse. La réalisation du test a été précédée par l'instruction et des exemples facilitant la démarche proposée. Le TJGTL englobait respectivement : I'article, l'adjectif possessif, la préposition, le COD/COI et la concordance des temps. Au total, on a prévu 20 doublets de phrases quatre pour chaque bloc grammatical. Les problèmes grammaticaux choisis ont été motivés par leur fréquence dans le langage courant et par les difficultés que les étudiants polonophones avaient éprouvées dans leurs productions libres pendant les cours de conversation ou de grammaire. Les participants avaient à disposition la version sonore des phrases, ainsi que de la version graphique. La décision d'offrir aux participants cette " double modalité » était motivée par la volonté de ne favoriser aucun groupe d'étudiants (ni les visuels, ni les auditifs). Le temps total consacré à la réalisation de ce test était de 3 minutes 45 secondes. On a effectué, dans l'ordre, le test basé sur la pression du temps, puis celui sans pression temporelle.

\subsection{Le test de jugement de grammaticalité en temps illimité}

Le test de jugement de grammaticalité en temps illimité (TJGTI) renvoie aux connaissances explicites en grammaire dont le locuteur est conscient et qu'il est capable, au moins avec un métalangage modeste, d'expliciter. Autrement dit, il s'agit des capacités métalinguistiques indispensables pour porter attention aux aspects formels de la langue (phonologiques, morpho-syntaxiques, lexicaux) que le locuteur natif commence à acquérir avec ses premières activités de lecture (Gombert, Gaux, Demont, 1994: 61) et auxquelles le locuteur adulte renvoie souvent au cours de son apprentissage d'une $L 2$ en exploitant ses connaissances en $\mathrm{L} 1$ et en cherchant consciemment ou à son insu les analogies entre deux systèmes linguistiques (Derkx 2005: 295). Certains didacticiens (Cuq 2003 : 127) associent même ces deux types de connaissances en les opposant aux connaissances implicites. On admet que les connaissances explicites demandent du temps, des efforts et de l'attention. Elles sont donc plus coûteuses sur le plan cognitif que les connaissances implicites, et elles sont plus rapidement sources de fatigue. Dans le domaine du langage, on a affaire à la conscience métalinguistique du locuteur dont découle la réflexion sur la langue conduisant aux comportements plus " mûrs » et au contrôle intentionnel des productions langagières.

\footnotetext{
${ }^{3}$ Le test dont on parle a été mené auprès de deux groupes : celui de niveau $A 2$, analysé dans le présent article, et un groupe B2 que nous ne prenons pas en considération dans cette analyse en raison d'autres objectifs formulés au préalable.
} 
Etant donné les caractéristiques des connaissances explicites, le TJGTI se pratique, comme l'indique son nom, sans limitation du temps et consiste, tout comme son correspondant en temps limité, à évaluer les phrases du point de vue de leur grammaticalité/agrammaticalité. Le plus souvent, le TJGTI accompagne le test effectué en temps limité. De plus, il contient les mêmes phrases et constructions. Le chercheur dispose donc d'un accès facile et rapide aux données qui l'intéressent. La réalisation de ces deux tests dans le même groupe peut, par conséquent, servir à comparer le niveau des connaissances garantissant les comportements langagiers rapides et la maitrise des connaissances favorisant le contrôle et l'autogestion linguistiques. II est important de noter que l'ordre de ces deux tests ne doit en aucun cas être arbitraire : le premier à mener est le TJGTL, pendant que le TJGTI vient en second.

Dans les recherches présentées dans les ouvrages didactiques, les phrases à évaluer sont, dans la plupart des cas, affichées sur l'écran de l'ordinateur ou imprimées sur des feuilles individuelles. II faut aussi observer que certains auteurs (Ellis 2009 : 46 ; Burston 2008 : 1794) demandent également aux participants d'indiquer le degré de certitude avec lequel ils ont formulé leur jugement. Ellis se sert d'une échelle allant de 0 à $100 \%$, ce qui lui permet de constater s'il $s^{\prime}$ agit de la connaissance de la règle de grammaire ou si la réponse est basée sur le sentiment (ang. rule vs feel). Burston, pour sa part, propose une gamme de quatre possibilités: (1) correcte, (2) probablement correcte, (3) probablement incorrecte et (4) incorrecte. Cette gamme lui est utile pour faire la différence entre jugement de grammaticalité et jugement d'acceptabilité, ce qui renvoie à une distinction entre la grammaire normative qui cherche à décrire une langue à partir de règles strictes et la grammaire descriptive dont l'objectif est de décrire la manière dont les gens parlent, ainsi que l'usage réel d'une langue. II est important de remarquer que dans le cas des apprenants peu avancés, le choix des phrases est (ou devrait être) limité à celles représentant la grammaire normative et contenant des règles strictes qui n'ouvrent pas l'espace au "flou " auquel chaque langue naturelle est condamnée (Yaguello 2015 : 79). On retrouve aussi des observations pertinentes à ce propos chez Burston (2008:1796) qui note que « la longueur de la phrase et le choix du vocabulaire peuvent être des sources de variabilité » et qu'il serait donc préférable que la longueur des phrases soit raisonnable et, en adaptant des phrases produites par des étudiants de groupes moins avancés, à ce que le vocabulaire soit usuel ou connu des sujets.

Le TJGTI, en tant qu'instrument mesurant les connaissances en langue, respecte les différences individuelles au niveau du temps que chaque participant nécessite pour traiter les informations. On peut aussi supposer que le manque de pression du temps favorise le calme et le sentiment de certitude, ce qui permet à chacun de travailler à son rythme. 
En dehors des qualités ici énumérées qu'il partage avec le TJGTL (accès facile aux structures grammaticales choisies par le chercheur, caractère formatif), le TJGTI comporte toutefois d'importantes contraintes.

La première concerne la formulation de la consigne qui reste trop complexe et contient des indices de subjectivité. Demander aux participants de juger des phrases et de présenter parallèlement la certitude avec laquelle le jugement a été fait semble être une tâche qui apporte au chercheur des informations pertinentes, mais la manière dont les participants sont sollicités révèle des indices de subjectivité. Ni l'estimation exprimée en pourcentage, ni la gamme de quatre possibilités n'apportent de réponses valables. Dans le premier cas, l'échelon est trop large et son choix peut être quelque peu aléatoire. Dans le deuxième cas, l'évaluation renvoie à la prise de conscience de ses propres processus cognitifs, ce qui dépasse la mesure des connaissances explicites en langue et entre sur le terrain de la conscience de soi et de la métacognition. Demander aux participants de formuler la règle conformément à laquelle ils ont jugé la phrase serait, à notre sens, une solution plus valable et présentant des indices d'objectivité. Si le chercheur tient à recevoir l'information concernant la connaissance des règles et ne se contente pas seulement du jugement fait par le participant, il peut aussi insérer dans la recherche un test des connaissances métalinguistiques (ang. metalinguistic knowledge test) qui vise à connaître les connaissances explicites de règles de $L 2$ explicitées avec un métalangage plus ou moins précis qui valide ou non le jugement (Elder 2009).

Les réponses qui résultent de la décision de « deviner » ou de répondre au hasard sont une autre limite du TGJTI. Ici, comme dans le TJGTL, il est impossible d'exclure que les participants ne prennent la décision sans connaître la règle. Une solution pourra consister, comme dans le cas du TJGTL, à offrir aux participants la possibilité d'indiquer leur doute ou leur manque de connaissance par un point d'interrogation.

Dans le TJGTI réalisé par nos soins, les participants ont été priés d'apporter leur jugement sans présenter leur degré de certitude. Le test en temps illimité comportait les mêmes doublets de phrases que le test en temps limité. Ce test n'avait que sa version graphique. Quant à la durée du TJGTI mené dans notre recherche, il faut remarquer que l'on n'a pas compté le temps dont chacun avait eu besoin, en admettant que cette information n'apporte pas de données ayant une influence sur les réponses formulées au préalable. On s'est limité à compter le temps du groupe en entier qui, dans notre cas, était de 7 minutes 20 secondes.

Dans la partie suivante, sont présentés les résultats de la recherche sur les connaissances implicites et explicites en FLE issus des tests de jugement de grammaticalité. 


\section{3. Étude empirique}

\subsection{Présentation du problème de recherche}

Le problème central pour la recherche a été d'estimer les connaissances implicites et explicites en grammaire des étudiants polonophones apprenant le FLE dans le cadre des études universitaires ${ }^{4}$. La recherche a été axée sur deux questions qui prenaient la forme suivante :

Q1 : Quel est le niveau des connaissances implicites et explicites en grammaire dans le groupe d'étudiants en FLE au niveau A2 ?

Q2 : Est-ce qu'il existe des rapports entre les connaissances implicites et explicites en grammaire dans le groupe examiné ?

La réponse à la première question avait pour objectif de fournir des informations portant sur le niveau de compétences des étudiants en termes des structures grammaticales considérées comme celles qui sont indispensables pour acquérir le niveau $\mathrm{A} 2$. Cependant, la réponse à la deuxième question permettait de constater si le développement des connaissances implicites et explicites en langue dans le contexte des études philologiques tend à l'équilibre ou bien s'il est plutôt orienté vers un type de connaissances.

\subsection{Participants à la recherche}

Le groupe d'étudiants qui a participé à la recherche représentait le niveau A2. Il venait de l'Université de Szczecin et avait suivi, au moment de la réalisation de la recherche, environ 300 heures de grammaire et de conversation. Le groupe comptait dix-neuf personnes dont quinze étaient de sexe féminin et quatre de sexe masculin. En dehors du français, les étudiants apprenaient aussi, dans le cadre de leurs études universitaires, une autre langue romane : italien ou espagnol au choix.

\subsection{Le déroulement de la recherche et les outils}

L'étude a été réalisée en deux étapes. La première étape consistait à juger la grammaticalité/agrammaticalité des 20 doublets de phrases dans un temps limité à 3 secondes par doublet ; dans un second temps, il convenait d'apporter

\footnotetext{
${ }^{4}$ La nature et le rôle des connaissances explicites et implicites en L2, ainsi que les résultats de la recherche sur la construction de celles-ci ont été le sujet de l'article séparé (voir plus W. Piegzik 2019).
} 
son jugement sur les mêmes doublets de phrases en temps illimité. Pour la première étape, on s'est servi du TJGTL et, pour la seconde du TJGTI.

La réalisation de l'étude en deux étapes a permis de :

- saisir et comparer le comportement langagier des étudiants dans les tâches rapides et dans les tâches sans limitation du temps,

- saisir les acquis et les difficultés des étudiants au niveau A2 pour prévoir une démarche de remédiation éventuelle,

- démontrer la validité des outils de recherche : le TJGTL et le TJGTI, et, si nécessaire, proposer les pas pour éliminer les insuffisances,

- vérifier si l'opinion courante sur le fait que les études universitaires ne privilégient pas l'aspect utilitaire de la langue et qu'elles se concentrent avant tout sur les connaissances explicites retrouve sa confirmation dans les résultats de la recherche.

\subsection{Présentation des résultats obtenus}

L'étude empirique réalisée à partir des tests de jugement de grammaticalité permet de constater que les connaissances implicites des étudiants en FLE au niveau A2 atteignent un taux de $60,8 \%$ ( 12,16 points sur 20 points au maximum) tandis que les connaissances explicites obtiennent un taux de $63 \%$ (12,61 points sur 20 points au maximum). Le niveau obtenu peut donc être considéré comme très similaire : le décalage entre les deux taux est de 0,45 point. La médiane pour les connaissances implicites est à 11 points et pour les connaissances explicites à 13 points. On observe des résultats différents au niveau du mode : dans le TJGTL, il est de 13 points et dans le TJGTI, il équivaut à 10 points. La valeur de l'écart-type (ang. standard deviation) obtenu est, pour le TJGTL à 2,64 et, pour le TJGTI, à 3,46. Les deux valeurs de l'écart-type permettent de constater que le groupe participant à la recherche était plutôt homogène, ce qui signifie que dans le groupe en question le nombre d'étudiants dont le niveau de maîtrise en langue dépassait de façon significative le niveau moyen n'était pas important (Wilczyńska et Michońska- Stadnik 2010 : 195-196). Etant donné les valeurs des indicateurs de tendance centrale obtenus et présentés plus haut, nous nous autorisons à formuler la première conclusion, à savoir que les étudiants participant à la recherche, mis dans une situation de réaction linguistique rapide et de réaction sans limitation du temps, obtiennent des résultats très similaires, sinon identiques. Les histogrammes cidessous représentent les résultats obtenus. Sur l'axe $X$ on met le nombre de points, sur l'axe $Y$, on représente la répartition des résultats obtenus. 

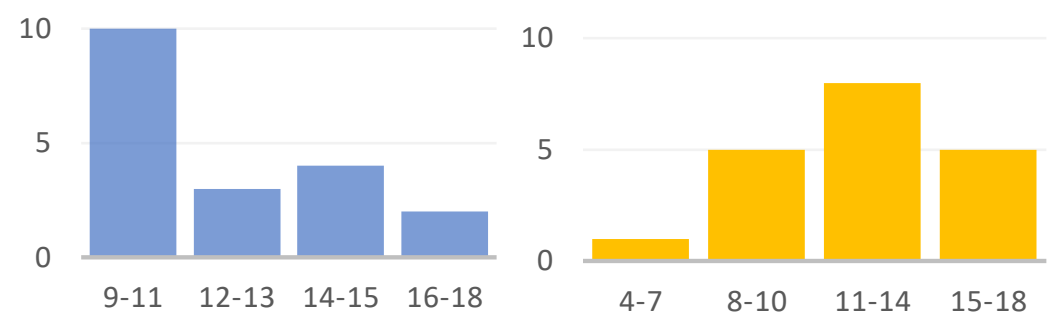

Figure 1 : Histogramme de répartition dans le TJGTL Figure 2 : Histogramme de répartition dans le TJGTI

Il est important de noter que, dans le cas des réactions en temps limité, la plupart des étudiants obtiennent un résultat dans l'intervalle 9-11 points, donc aux alentours de la médiane. Peu nombreux sont les étudiants aux résultats les plus hauts, 16-18 points. Aucun étudiant n'a obtenu le résultat maximum. Cela peut être dû au fait que les quatre dernières questions incluaient le matériel signalé en cours de grammaire, mais qui n'a pas été mis en pratique. Dans le cas des réactions en temps illimité, la répartition des résultats est différente. Les résultats dominants sont ceux qui s'inscrivent dans l'intervalle 11-14 points. Personne n'a eu le résultat maximum et un étudiant a obtenu un score particulièrement bas (4 points seulement), au-dessous de toutes les valeurs obtenues ${ }^{5}$.

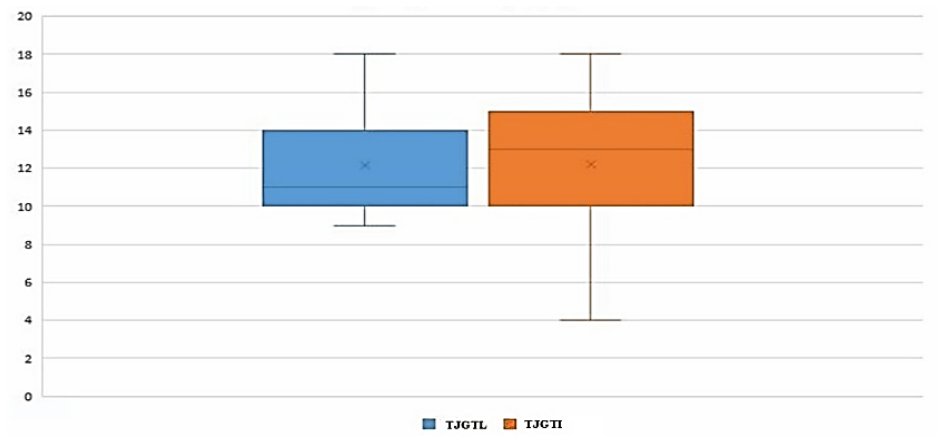

Figure 3 : Diagrammes en boîtes à moustache illustrant les tendances centrales dans le groupe $\mathrm{A} 2$

\footnotetext{
${ }^{5}$ Etant donné que le groupe était peu nombreux et que la recherche présentée servait de recherche de pilotage, nous avons décidé de ne pas nous référer à la pratique qui consiste à couper le résultat le plus bas et le plus élevé pour éliminer les extrémités. Nous sommes en même temps consciente que cette pratique peut être pertinente surtout lorsque l'objectif principal est de découvrir les tendances centrales dans des groupes nombreux et d'étendre les résultats obtenus à toute la population.
} 
En analysant plus en détail les tendances centrales présentes dans le groupe, nous nous référons aux diagrammes en boîtes à moustache qui nous permettent d'analyser la répartition des résultats obtenus en tenant simultanément compte de cinq indicateurs : médiane, premier et troisième quartile, résultat minimal et résultat maximal.

La première boîte représentant les résultats du TJGTL, allant du premier quartile $(Q 1=10)$ au troisième quartile $(Q 3=14)$, représente $75 \%$ des résultats obtenus dans le test. La médiane qui coupe la boîte découvre l'asymétrie au niveau de la distribution des données obtenues dans ce test : la plupart des résultats se situent au-dessus du résultat qui est à 11 points. La moustache supérieure signifiant le résultat maximal $(r \max =18)$ et la moustache inférieure renvoyant au résultat minimal $(r \min =9)$ montrent l'étendue qui, au niveau des réactions rapides des étudiants, est à 9 points.

La deuxième boîte, illustrant de façon analogique les résultats du TJGTI, présente la valeur du premier quartile $(Q 1=10)$ et celle du troisième quartile $(Q 3=15)$ en découvrant une tendance centrale aux résultats légèrement plus élevés. Avec la médiane qui coupe la boîte en deux moitiés, on observe que les résultats au-dessous de la médiane dominent et produisent une asymétrie. Il est à noter aussi que l'étendue dans le TJGTI, égale à 14 points, est beaucoup plus importante que dans le TJGTL. Cela peut mener à la conclusion que les connaissances explicites en grammaire dans le groupe participant à la recherche ne sont pas suffisamment structurées.

Pour répondre à la question sur les rapports entre les connaissances implicites et explicites dans notre groupe, nous nous sommes référée aux statistiques inductives et, dans le cadre de ces statistiques, à la mesure largement utilisée par les chercheurs dont les groupes sont peu nombreux, à savoir le coefficient de corrélation de Pearson ( $r$ de Pearson) qui est une mesure de corrélation entre deux variables et qui se situe entre +1 et -1 . La valeur obtenue pour notre groupe $r=0,676451$ (avec $p<0,05$ ) nous autorise à constater que la corrélation entre les connaissances implicites et explicites dans le groupe d'étudiants en FLE au niveau A2 est modérée.

\section{Conclusion}

Toutes les données obtenues au cours de la recherche nous permettent de conclure que le niveau des connaissances implicites et explicites des structures grammaticales testées dans le groupe d'étudiants en FLE au niveau A2 atteint des taux similaires dont la différence ne dépasse pas un point. En outre, les connaissances implicites et explicites des étudiants se développent 
de façon équilibrée. Malgré cet équilibre au sein des deux types de connaissances, on observe des irrégularités importantes et une corrélation modérée. Il est également important de souligner que les taux de réussite se situant au niveau de $60 \%$ pour les connaissances implicites et au niveau de $63 \%$ pour les connaissances explicites peuvent sembler peu satisfaisants tant pour les enseignants que pour les étudiants, d'autant plus que les tests se limitaient, au moins dans la majorité, aux structures de base attribuables au niveau A2.

De notre recherche résulte aussi que les tests de jugement de grammaticalité nous ont permis de relever et de mesurer les difficultés éprouvées par les étudiants polonophones lors de leur apprentissage-acquisition du FLE, même si le repérage et la mesure concernent un nombre restreint de problèmes grammaticaux. Ce sont les articles et les prépositions, qui en tant que blocs grammaticaux choisis, se sont avérés les moins " forts " et qui demandent davantage de travail. Cela concerne tant les réactions rapides que celles sans limitation du temps. De toute évidence, il serait intéressant de répéter les deux tests dans le même groupe d'étudiants en proposant les mêmes blocs grammaticaux ou en se concentrant sur un seul bloc choisi (p.ex. bloc consacré aux articles). Cette démarche pourra apporter un aperçu plus approfondi sur les connaissances en grammaire des étudiants et répondre aux questions de recherche plus en détail. Elle pourra aussi apporter des données importantes sur le facteur lié aux "réponses au hasard ", lequel s'inscrit dans la nature des outils présentés et constitue l'une des limites importantes.

En outre, on a observé que l'entrainement aux comportements spontanés tel que le TJGTL peut susciter, au moins chez certains des étudiants prenant part à cette recherche, une réflexion métalinguistique et une autoévaluation. Il peut donc, en dehors de sa fonction essentielle (collecte de données), servir au développement de la métacognition qui, comme l'avance Jacques Tardif (1992: 58), " est une caractéristique distinctive entre les experts et les novices ". Même si les deux instruments utilisés dans l'étude empirique se limitent à des capacités qui n'exigent pas de production langagière, ils fournissent des informations qui peuvent être complémentaires à celles issues des études orientées vers les habilités de production libre. Du point de vue du chercheur, il sera important d'utiliser ces tests conjointement avec d'autres instruments et de veiller à surmonter les insuffisances décrites dans la présente analyse (voir 2.1 et 2.2). Tout en restant conscient des limites des tests de jugement de grammaticalité, il est important de noter que le chercheur qui se décide à s'en servir a des chances non seulement de collecter des données pertinentes, mais aussi d'inspirer chez les sujets une réflexion métalinguistique grâce à laquelle leur apprentissage-acquisition renforcé par la 
Évaluer les connaissances implicites et explicites en français langue étrangère par...

prise de conscience peut aller de l'avant en explorant les nouvelles possibilités que chaque langue offre à ses locuteurs.

\section{BIBLIOGRAPHIE}

Bajrić S. (2005), Question d'intuition. Langue française No 147, p. 7-18. Online : http://www.persee.fr/doc/lfr_0023-8368_2005_num_147_1_6860 [accès : 20.05.2020].

Barth B.-M. (2002), Le savoir en construction. Paris : Retz.

Burston M. (2008), Évaluer la compétence par un test de jugement de grammaticalité : le cas de la relativisation chez des apprenants chypriotes hellénophones avancés en FLE, in : Durand J. Habert B., Laks B. (réd.), Collection des Congrès Mondiaux de Linguistique Française, Paris, France 2008, 1791-1803. Online : https://www.researchgate.net/publication/ 45069247_Evaluer_la_competence_par_un_test_de_jugement_de_gr ammaticalite_le_cas_de_la_relativisation_chez_des_apprenants_chyp riotes_hellenophones_avances_en_FLE [accès : 09.11.2019].

Chomsky, Noam. (1965). Aspects of the Theory of Syntax. Cambridge : Massachusetts : MIT Press.

Cuq J.-P. (réd.) (2003), Dictionnaire de didactique du français langue étrangère et seconde. Paris : Cle International.

Derkx V. (2015), L'intuition en sciences du langage : de l'énonciation à la grammaticalité des énoncés. Thèse de doctorat en linguistique non publiée. Paris.

Dubois J. (réd.), (2012), Dictionnaire de linguistique et des sciences du langage. Paris : Larousse.

Gombert J.E., Gaux, Ch., Demont, E. (1994), Capacités métalinguistiques et lecture, quels liens? (in) Repères. Recherches en didactique du français langue maternelle $\mathrm{nr} 9$, p. 61-73. Online : https://www.persee.fr/doc/ reper_1157-1330_1994_num_9_1_2111 [accès: 09.11.2019].

DeKeyser R.M. (2005), Implicit and explicit learning (in) : C.J. Doughty, M.H. Long (réd.), The Handbook of Second Language Acquisition. Oxford: Blackwell, p. 313-348.

Elder C. (2009), Validating a Test of Metalinguistic Knowledge (in) R. Ellis, S. Loewen, C. Elder, R. Erlam, J. Philp \& H. Reinders (réd.), Implicit and Explicit Learning, Knowledge and Instruction. Bristol: Multilingual Matters, p. 113-138.

Ellis, R. (2009), Implicit and Explicit Learning, Knowledge and Instruction (in) R. Ellis, S. Loewen, C. Elder, R. Erlam, J. Philp \& H. Reinders (réd.), Implicit and Explicit Learning, Knowledge and Instruction. Bristol: Multilingual Matters, p. 3-25. 
Han Y. (2000), Grammaticality judgment tests: How reliable and valid are they? (in) "Applied Langauge Learning" nr 11(1), p. 177-204.

Kim M. (2010), The effects of storytelling on adults English language learners (in) "Linguistic Research" nr 27 (3), p. 447-473.

Loewen C. (2009), Grammaticality Judgment Test and the Measurement of Implicit and Explicit L2 Knowledge (in) R. Ellis, S. Loewen, C. Elder, R. Erlam, J. Philp \& H. Reinders (réd.), Implicit and Explicit Learning, Knowledge and Instruction. Bristol : Multilingual Matters, p. 94-112.

Nadeau M., Fisher C. (2011). Les connaissances implicites et explicites en grammaire : quelle importance pour l'enseignement ? Quelles conséquences? (in) „Bellaterra Journal of Teaching \& Learning Language \& Literature" Vol. 4 (4), p. 1-31. Online : https://www.researchgate.net/ publication/277225020_Les_connaissances_implicites_et_explicites_e n_grammaire_quelle_importance_pour_l'enseignement_Quelles_con sequences [accès: 09.11.2019].

Piegzik W. (2019), La construction des connaissances implicites et explicites en langue étrangère : vers un développement harmonieux ou déséquilibré ? Cas des étudiants polonophones étudiants en FLE développant le plurilinguisme (in) : M. C. Ainciburu (réd.), En camino hacia el plurilingüismo. Actos del IV Congreso Internacional Nebrija en Linqüística Aplicada a la Enseñanza de Lenguas, Madrid : Universidad Nebrija, 163-174. Online : https://www.nebrija.com/vida_universitaria/servicios/pdfpublicaciones/ ActasIVCongresoSLANebrija.pdf [accès: 09.01.2020].

Polański K. (red.) (2003), Encyklopedia językoznawstwa ogólnego, Wrocław: Ossolineum.

Tardif J. (1992), Pour un enseignement stratégique : apport de la psychologie cognitive. Montréal : Les Éditions Logiques.

Wilczyńska W., Michońska-Stadnik A. (2010), Metodologia badań w glottodydaktyce. Wprowadzenie. Kraków: Avalon.

Yaguello M. (1988), Catalogue des idées reçues sur la langue. Paris : Éditions du Seuil. 


\section{Annexe 1}

\section{Test de jugement de grammaticalité basé sur le temps limité}

Exercice : Écoutez une seule fois les phrases proposées, puis soulignez la phrase qui est, à votre sens, correcte. Vous disposez d'un temps limité, concentrez-vous bien.

\begin{tabular}{|c|c|c|}
\hline $\mathrm{Nr}$ & Phrase 1 & Phrase 2 \\
\hline 1. & Ce n'est pas de pain. & Ce n'est pas du pain. \\
\hline 2. & J'aime travailler avec la groupe A. & J'aime travailler avec le groupe A. \\
\hline 3. & Tu as vu la clé voiture ? Je ne la trouve pas. & Tu as vu le clé voiture ? Je ne le trouve pas \\
\hline 4. & Y a-t-il les vols directs pour Boston ? & Y a-t-il des vols directs pour Boston? \\
\hline 5. & Ills pensent à leurs enfants. & Ills pensent à ses enfants. \\
\hline 6. & Mon histoire est intéressante. & Ma histoire est intéressante. \\
\hline 7. & Les parents écrivent souvent à leur amis. & Les parents écrivent souvent à leurs amis. \\
\hline 8. & Prête-moi tes pantalons! & Prête-moi ton pantalons! \\
\hline 9. & Elle a choisi à quitter la ville. & Elle a choisi de quitter la ville. \\
\hline 10. & Ill s'est mis à pleuvoir. & II s'est mis de pleuvoir. \\
\hline 11. & Mes parents habitent au Portugal. & Mes parents habitent en Portugal. \\
\hline 12. & Ils ont été à Bruxelles. & Ils ont été aux Bruxelles. \\
\hline 13. & Vous nous avons dit la vérité. & Vous nous avez dit la vérité. \\
\hline 14. & Je l’ai aidé à faire le ménage. & Je lui ai aidé à faire le ménage. \\
\hline 15. & Nous les voulons voir ce soir. & Nous voulons les voir ce soir. \\
\hline 16. & Ne lui téléphone pas demain! & Ne téléphone-lui pas demain! \\
\hline 17. & J’ai lu le livre que tu m'as offert. Excellent! & J'ai lu le livre que tu m’avais offert. Excellent! \\
\hline 18. & Quand je suis arrivée, il avait déjà préparé le dîner dîner. & Quand je suis arrivée, il préparait déjà le dîner. \\
\hline 19. & II faut que vous serez à temps. & II faut que vous soyez à temps. \\
\hline 20. & Je pense que tu réussisses. & Je pense que tu réussiras. \\
\hline
\end{tabular}

